NP,IT, European Research Conference on Fundamental Aspects of Clusters, Elounda, Crete, Greece, May 26-June 1. 1993

\title{
COLLISIONS OF MOLECULES WITH CLUSTERS: A QUASICLASSICAL STUDY*
}

\author{
J. Jellinek and Z. B. Güvenç \\ Chemistry Division \\ Argonne National Laboratory \\ Argonne, IL 60439, USA
}

\section{DISCLAIMER}

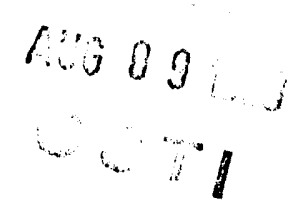

\begin{abstract}
This report was prepared as an account of work sponsored by an agency of the United States Government. Neither the United States Government nor any agency thereof, nor any of their employees, makes any warranty, express or implied, or assumes any legal liability or responsibility for the accuracy, completeness, or usefulness of any information, apparatus, product, or process disclosed, or represents that its use would not infringe privately owned rights. Reference herein to any specific commercial product, process, or service by trade name, trademark, manufacturer, or otherwise does not necessarily constitute or imply its endorsement, recommendation, or favoring by the United States Government or any agency thereof. The views and opinions of authors expressed herein do not necessarily state or reflect those of the United States Government or any agency thereof.
\end{abstract}

*Work performed under the auspices of the Office of Basic Energy Sciences, Division of Chemical Science, US-DOE under contract number W-31-109-ENG-38.

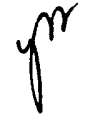




\title{
COLLISIONS OF MOLECULES WITH CLUSTERS: A QUASICLASSICAL STUDY
}

\author{
J. Jellinek and Z. B. Güvenç \\ Chemistry Division \\ Argonne National Laboratory \\ Argonne, IL 60439, USA
}

\section{INTRODUCTION}

Among the most intriguing and fascinating aspects of the physico-chemical phenomena taking place in very different systems, which form the subject matter of disciplines as diverse as nuclear physics, atomic physics, physics and chemistry of individual molecules, of atomic and molecular clusters, and even of liquids and solids (each of these has welldefined limits of applicability), are those that cross the interdisciplinary boundaries and are common for the different systems. Unearthing universality is one of the central objectives of a scientific pursuit not only because universal means fundamental, but also because universality introduces a unifying and often simplifying order into our picture of the physical reality.

Atomic and molecular clusters were utilized first as convenient auxiliary objects for microscopic studies of the bulk and surface properties of the condensed phase of matter. The recent surge in the scope and intensity of the cluster research is, however, attributable even more to the recognition that clusters play a special role as a new state of matter. This state fills the gap between gases, on the one hand, and liquids and solids, on the other. The practical importance of the cluster studies becanne well-appreciated as well: clusters play a central role in a variety of natural phenomena (e.g., in the upper atmosphere) and in processes of environmental concern, as well as in technologies related to heterogeneous catalysis, microelectronics, materials design and fabrication, etc. Although broad and interdisciplinary, as judged by the traditional divisions in physics and chemistry, cluster research is establishing itself as a sovereign field with its own subject(s), methodologies and objectives 1 . One of the major objectives is to understand the evolution of the different physico-chemical properties of clusters with their size, i.e., the number of atoms and/or molecules in a cluster, and to unravel the mechanisms of this evolution in different materials as defined by the nature of the corresponding interatomic and intermolecular interactions. Viewed as n-body systems, atomic and molecular clusters and many of the cluster-related phenomena have much in common with atomic nuclei (clusters of nucleons) 
and the phenomena studied by nuclear physics. This is true notwithstanding the fact that the strength and range of forces acting between atoms are very different from those characterizing atomic nuclei. It is not an accident that concepts such as the liquid drop model, shell structure, magic numbers and others, originally introduced by nuclear physicists, have been adopted by and utilized fruitfully in the cluster field 2 . Problems and phenomena related to the shape (structure), stability, fusion and fission are common for both, clusters and nuclei. The similarities between the cluster radioactivity in nuclei ${ }^{3}$ and the fragmentation patterns of atomic clusters is striking. Even such a new subject as superdeformations in high-spin nuclei has its cluster analog. The structural and dynamical effects of high angular momenta in clusters have been analyzed recently ${ }^{4}$. Collision phenomena involving a cluster or a nucleus have especially many common aspects.

The processes induced by collisions of metal clusters with molecules have been and remain one of the central subjects of the experimental cluster research ${ }^{5}$. The interest in these is fueled primarily by their direct relevance to heterogeneous catalysis. The majority of the related theoretical studies are electronic structure calculations performed at fixed nuclear geometries ${ }^{6-8}$. In fact, many of these calculations ${ }^{7,8}$ were aiming at explori...g surface-molecule, rather than cluster-molecule, interactions and, as a consequence, the cluster geometries were represented by slabs of the appropriate lattices rather than by the corresponding energetically more favorable - for the cluster sizes considered - nonlattice structures. Only in a few theoretical studies have cluster-molecule systems been treated dynamically9-16. These studies utilize model or semiempirical potentials to describe the interaction between the atoms within the cluster and/or (in case the cluster is assumed to be rigid) between the cluster and the molecule. The goal is to understand and to characterize the mechanisms of the different processes that may follow a collision of a molecule with a cluster. These processes include molecular or dissociative adsorption, diffusion of the adsorbed species, their possible recombination and desorption, as well as direct scattering of the molecule from the cluster. The mechanisms governing these processes depend crucially on the details of the interaction potential. Therefore, a concurrent goal of the studies is to arrive at a better modelling of the potentials and to gain a deeper insight into the character of the interatomic interactions. A way to accomplish this is to compute quantities that can be measured experimentally', then to compare the calculated and the measured data (provided, of course, the latter are available), and to "tune" the parameters of the potential, if necessary, until an agreement between the two is achieved.

In what follows we present results of a simulation study of a collision of a $D_{2}$ molecule with a $\mathrm{Ni}_{13}$ cluster. The focus is, primarily, on the reactive channel of the interaction, i.e., on the dissociative adsorption of the molecule on the cluster. After a brief outline of the potentials used and of the computational procedure we present results on the energy dependence of the reaction, the possible correlation between the cluster structure and its reactivity, and the effect of the cluster temperature. The role of the indirect pathway to the reaction, which involves formation of transient states of molecular adsorption as precursors to eventual dissociative adsorption, is examined. A novel procedure is utilized to calculate the probabilities of formation of these cluster-molecule complexes ("resonances") and their lifetimes. The correlation between the calculated and the available measured data is discussed, and an example of tuning the cluster-molecule interaction potential is given. 


\section{THEORETICAL BACKGROUND AND METHODOLOGY}

The time evolution of the $\mathrm{Ni}_{13}+\mathrm{D}_{2}$ collision system is generated by solving Hamilton's equations of motion for all the atoms. The forces acting on the atoms are calculated from the potential energy function $V$,

$$
\mathrm{V}=\mathrm{V}_{\mathrm{EA}}+\mathrm{V}_{\text {LEPS }} \text {, }
$$

where $V_{E A}$ is a so-called embedded-atom fotential describing the interaction between the $\mathrm{Ni}$ atoms, and $\mathrm{V}_{\text {LEPS }}$ is a LEPS (London-Eyring-Polanyi-Sato) function representing the $D-D$ and the $D_{2}-N_{13}$ interactions. We use Voter and Chen's ${ }^{17}$ parameterization of $V_{E A}$, which is based on fitting not only bulk properties of nickel but also those of Ni2. As a consequence, one may expect this potential to be more adequate for clusters. In our original calculations we used $V_{\text {LEPS }}$ in the form of the potential energy surface (PES)Il of Raghavan et al., 9 which we modified by a smoothing function of the same form as used by

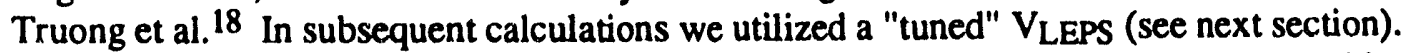

The initial conditions were specified as follows. The $\mathrm{D}_{2}$ molecule was prepared in a given quantized vibrational $v_{i}$ and rotational $j_{i}$ state using a quasiclassical prescription ${ }^{19}$. The molecule was placed "asymptotically far" from the cluster (separation of $8.5 \AA$ between the corresponding centers of mass) and sent towards the cluster with a given collision (i.e., relative translational) energy $E_{t}$ and impact parameter $b$. The initial state of the cluster was characterized by its structure and temperature $\mathrm{T}$ defined as

$$
T=\frac{2\left\langle E_{k}\right\rangle}{(3 n-6) k}
$$

where $E_{k}$ is the internal kinetic energy of and $n$ is the number of atoms in the cluster, $k$ is the Boltzmann constant, and $<>$ denotes time-average. The initial total linear and angular momenta of the cluster were chosen to be zero.

Hamming's modified fourth-order predictor-corrector integrator was used to generate the phase space trajectories. The maximal step-size of $(0.5-1) \times 10^{-15} \mathrm{~s}$ assured conservation of the total energy and total linear and angular momenta within $0.03-0.15 \%$. Batches of $N=500-10000$ trajectories were run for each set of initial conditions. These differed by the initial phase of the $\mathrm{D}_{2}$ oscillator, the initial coordinates and momenta of the cluster atoms corresponding to a fixed total energy of the cluster, and the initial relative orientation of the molecule and of the cluster. Each trajectory was run until one of the two events took place: (1) the molecule dissociated on the cluster, (2) after interaction with the cluster, the molecule departed from it back into the "asymptotic region". The trajectory was deemed to be reactive and was terminated when and if the distance between the $D$ atoms reached the value of $2.223 \AA$, which is three times the equilibrium bond length of the $D_{2}$ molecule. The trajectory was qualified as nonreactive when and if the $D_{2}$ molecule returned to the asymptotic region. (Of course, were the trajectories run long enough, they all would emerge as nonreactive because of the nonzero probability for the $D$ atoms to recombine and for the molecule to desorb.) The state-resolved probabilities $P_{v_{i}, j_{i}, T_{1} . . .}\left(E_{t r}, b\right)$ of the different processes were calculated as

$$
P_{v_{i}, j_{i}, T, \ldots}\left(E_{t r}, b\right)=\frac{\tilde{N}_{v_{i} j_{j}, T}, \ldots\left(E_{t r}, b\right)}{N}
$$

where $\tilde{\mathrm{N}}$... is the number of trajectories in a batch that resulted in the process of interest. The cross sections $\sigma . . .\left(E_{\mathrm{tr}}\right)$ were computed using the formula 


$$
\sigma_{v_{i}, j, T, \ldots}\left(E_{t r}\right)=2 \pi \int_{0}^{b_{\max }} b P_{v_{i}, j_{i}, T, \ldots .}\left(E_{t r}, b\right) d b
$$

where $b_{\max }$ is the largest impact parameter contributing to the process of interest. The rate constants $k . . .\left(T_{t r}\right)$ were obtained using the expression

$$
k_{v_{i} j_{i}, T_{,} . . .}\left(T_{t r}\right)=\pi \mu\left(\frac{2}{\pi \mu k T_{t r}}\right)^{3 / 2} \int_{0}^{\infty} \sigma_{v_{i} j_{i}, T, \ldots}\left(E_{t r}\right) E_{t r} \exp \left(-\frac{E_{t r}}{k T_{t r}}\right) d E_{t r}
$$

where $\mu$ is the reduced mass of the cluster-molecule system, and $T_{t r}$ is its translational ("collisional") temperatire.

Examination of the individual trajectories indicates that both the reactive and the nonreactive interactions of the molecule with the cluster may be direct or indirect. The latter involve formation of cluster-molecule complexes (resonances) which are transient states on the pathway to an eventual dissociation or desorption of the molecule. The important characteristics of the resonances are the probabilities (cross sections) of their formation and their lifetimes. These were calculated using the following approach. Let $P . . .\left(E_{t r}, b\right)$ be the probability of the direct reaction (i.e., of the dissociation of the molecule upon impact with the cluster) and $P^{I R} .\left(E_{t r}, b\right)$ - the probability of the indirect reaction. Alternatively, PIR. $\left(E_{t r}, b\right)$ is the probability of formation of reactive resonances, i.e., precursors to dissociative adsorption. If $\kappa_{\text {d... }}^{d}\left(E_{t r}, b\right)$ is the rate constant for dissociation of the adsorbed molecules, then the time-dependent probability $P^{R}$... $\left(E_{t r}, b \mid t\right)$ of the reaction (i.e., of the dissociative adsorption of the molecule) is

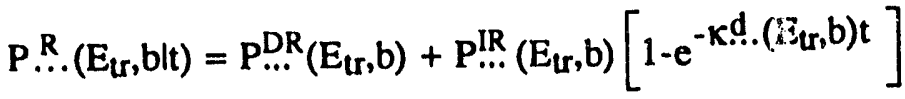

$$
\begin{aligned}
& =P_{\ldots}^{R}\left(E_{t r}, b\right)-P_{\ldots}^{I R}\left(E_{t r}, b\right) e^{-k d . .\left(E_{t r}, b\right) t},
\end{aligned}
$$

where

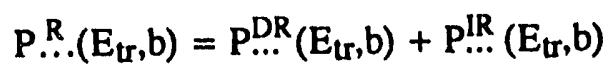

is the total probability of the reation, and the time $t \geq 0$ is measured from the "instant of arrival" $t_{a r}$ of the molecule at the cluster:

$$
t=t_{r}-t_{a r}, \quad t_{r} \geq t_{a r}
$$

In Eq. (8) $t_{r}$ is the running time of propagation of an individual trajectory. Times $t_{r}<t_{g r}$ correspond to the segment of the trajectory that represents the motion of the molecule towards the cluster. Viewing the dissociation of the molecule as an instantaneous process and assuming that $t_{a r}$ is a function of $E_{t r}$ and $b$ only i.e., neglecting the dependence of $t_{a r}$ on the initial phase of the $D_{2}$ oscillator, initial shape of the cluster and the initial relative orientation of the twoj we define $t_{a r}$ as that value of $t_{r}$ at which $P$... $\left(E_{t_{r}}\right.$, blt $\left.t_{r}\right)$ changes its value from zero to $P_{\ldots}^{D R}\left(E_{t r}, b\right)$. In case $P \ldots\left(E_{t r}, b\right)=0, t_{a r}$ is defined as the largest value of $t_{r}$ at which still $P^{R} .\left(E_{t r}, b \mid t_{r}\right)=0$. 
It follows from Eq. (6) that

$$
\ln \left[P \stackrel{R}{R}\left(E_{t r}, b\right)-P . . .\left(E_{t r}, b l t\right)\right]=-\kappa+.\left(E_{t r}, b\right) t+\ln P_{\ldots}^{l R}\left(E_{t r}, b\right)
$$

Computing the left-hand-side (lhs) of Eq. (9) on a grid of values of $t$ and fitting the results to a linear function one obtains $K \stackrel{d}{d}\left(E_{\mathrm{tr}}, \mathrm{b}\right)$ and $\mathrm{P}^{\mathrm{IR}},\left(\mathrm{E}_{\mathrm{tr}}, \mathrm{b}\right)$. The characteristic lifetime $\tau$...( $\left.E_{t r}, b\right)$ of the reactive resonances is defined as the inverse of $k ! .\left(E_{t r}, b\right)$. One can introduce a b-averaged rate constant $\bar{\kappa} d .\left(E_{t r}\right)$ and using Eq. (4) rewrite Eq. (9) in terms of the corresponding cross sections:

$$
\ln \left[\sigma \stackrel{R}{R}\left(E_{t r}\right)-\sigma . . .\left(E_{t r} t\right)\right]=-\bar{k} \cdot . .\left(E_{t r}\right) t+\ln \sigma_{\ldots}^{I R}\left(E_{t r}\right) .
$$

The time $t$ here is measured from an effective instant of arrival $\tilde{t}_{a r}$, which depends only on $E_{t r}$. The definition of $t_{a r}$ is similar to that of $t_{a r}$, only the probabilities have to be replaced by the corresponding cross sections. Computing the lhs of Eq. (10) on a grid of values of $t$ and fitting the results to a linear function one obtains $k . d .\left(E_{t r}\right)$ and the cross section of formation of reactive resonances $\delta$... $\left(E_{t r}\right)$. The b-averaged characteristic lifetime $\tau$...( $\left(E_{t r}\right)$ of these resonances is the inverse of $\bar{\kappa} \cdot . .\left(E_{t r}\right)$.

The definitions of $t_{a:}$ and $\tilde{t}_{\mathrm{ar}}$ given above involve two assumptions: (1) the instant of arrival depends on $b$ and/or $E_{t r}$ only, and (2) the dissociation of the adsorbed molecule is an instantaneous event. Although satisfied to a high degree of accuracy, these assumptions are approximations. As a consequence, a direct application of the definition of $\operatorname{tar}_{\mathrm{ar}}\left[\mathrm{t}_{\mathrm{ar}}\right]$ to a computed $P^{R} . .\left(E_{t r}, b \mid t_{r}\right)\left[\sigma^{R} .\left(E_{t r} t_{r}\right)\right]$ function may be somewhat ambiguous in cases when $P \ldots R\left(E_{t r}, b\right)\left[\sigma \ldots R\left(E_{t r}\right)\right]$ is zero or small. For a further discussion of this point and the resolution of the ambiguity we refer the reader to Ref. 15 . In cases when one is interested in the lifetimes of the resonances, but not in the probabilities (cross sections) of their formation, the determination of $t_{a r}\left[\tau_{a r}\right]$ may be bypassed. It is clear that fitting the lhs of Eqs. (9) and (10) to a linear function of $t_{r}$ (instead of $t$ ) does not alter the values of

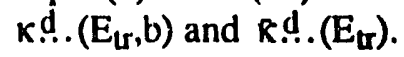

\section{RESULTS AND DISCUSSION}

Three geometries - icosahedral (ico), hexagonal close-packed (hcp), and cuboctahedral (cubo) - were considered as initial structures of the $\mathrm{Ni}_{13}$ cluster (Fig. 1). Whereas the cubo geometry is a fragment of the face-centered cubic lattice of the bulk nickel, the ico isomer is the most stable structure of $\mathrm{Ni}_{13}$ as determined from the embedded-atom potential.

A factor complicating the dynamical analysis of phenomena involving clusters is that one has to consider the simultaneous time evolution of a relatively large number of atoms or, alternatively, degrees of freedom. An additional complication arises from the fact that this time evolution may be the result of a number of different dynamical processes, which take place sequentially or simultaneously. It may prove exceedingly difficult, or even impossible, to extract the mechanisms of the incividual processes or their fine details directly from a large number of sizable numerical data sets. Computer generated visualization is a very efficient tool to analyze such data sets. It was through visualization that we first noticed transformation of the cubo and hcp geometries of $\mathrm{Ni}_{13}$ into its ico form as a consequence of a collision (either reactive or inelastic) of the cluster with a $D_{2}$ molecule. The cubo and hcp isomers of $\mathrm{Ni}_{13}$ are metastable and even a minor perturbation (such as accumulation of computer roundoff errors) causes their transformation into the ico form. 

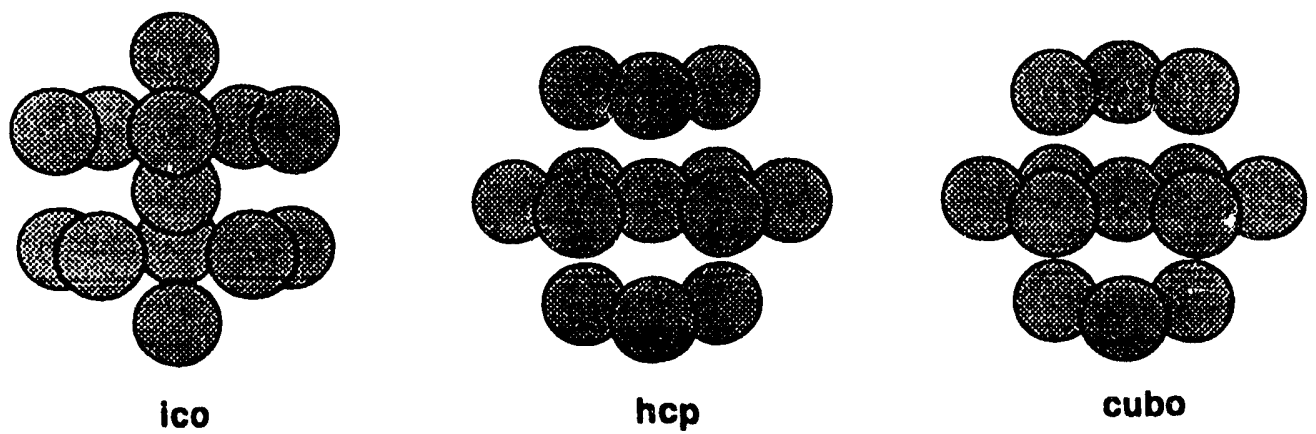

Figure 1. The icosahedral, hexagonal close-packed, and cuboctahedral structures of a 13-atom cluster. Their energies for $\mathrm{Ni}_{13}$, as defined by $\mathrm{V}_{E A}$, are $-41.12 \mathrm{eV},-39.53 \mathrm{eV}$, and $-39.51 \mathrm{eV}$, respectively.

More precisely, the structure of the cluster begins to oscillate between the cubo or hcp and the ico geometries. A "movie" of a collision of a $D_{2}$ in $\left(v_{i}=1, j_{i}=0\right)$ state with an ico $\mathrm{Ni}_{13}$ prepared at room temperature, which leads to a direct dissociation of the molecule, diffusion of the $\mathrm{D}$ atoms on the cluster surface, their recombination, and eventual desorption of the $D_{2}$, is shown in Fig. 2.

Details of the dynamical processes taking place can be displayed also by plotting distances, such as the D-D separation, and the separations between the individual D atoms, as well as the center of mass $(\mathrm{cm})$ of the $D_{2}$ molecule, on the one hand, and the $\mathrm{cm}$ of the cluster, on the other, as functions of time. An example for the case of a direct inelastic scattering is shown in Fig. 3. One notices that the vibrational motion of the molecule is almost not perturbed by the collision event that takes place at $t=7.3$ time units $\left(10^{-14} \mathrm{~s}\right)$. The molecule, however, acquires an additional rotational excitation that is signified by a decrease in the time interval between neighboring points at which the atom-cluster $(\mathrm{cm})$ distances cross. This time interval is equal to half of the rotational period.

The cross sections for the dissociative adsorption of $D_{2}$ on a room-temperature ico $\mathrm{Ni}_{13}$ are shown in Fig. 4 as functions of the collision energy for different initial rovibrational states of the molecule. The $\left(v_{i}=1, j_{i}=0\right)$ and $\left(v_{i}=0, j_{i}=10\right)$ states of $D_{2}$ have almost the same energy, and they were considered to test how the dissociative adsorption of the molecule depends on the mode of deposition of its initial energy. The graphs clearly display the following features: (1) the reactivity of the cluster increases monotonically with the collision energy; (2) larger cross sections correspond to higher initial rovibrational energies of the molecule; (3) the reaction is state-selective: the cross sections for $\left(v_{i}=1, j_{i}=0\right)$ are considerably higher than those for $\left(v_{i}=0, j_{i}=10\right) ;(4)$ in order for the reaction to take place the collision energy has to exceed a small $\left(v_{i}, j_{i}\right)$ dependent threshold value.

The corresponding cross sections for the reaction of the molecule with zerotemperature cubo and hcp $\mathrm{Ni}_{13}$ are shown in Fig. 5. Although these two structures of the cluster are metastable, one can still consider the reactivity of the cubo and hcp isomers of $\mathrm{Ni}_{13}$ because the reaction time is, in most cases, shorter than the characteristic time of a structural transition into the ico form. Whereas the reaction cross sections for the cubo and hcp isomers are very similar, they are different from those for the ico $\mathrm{Ni}_{13}$ both qualitatively and quantitatively. The ico isomer is generally less reactive. More striking are, however, the qualitative differences. The cross sections for the cubo and hcp structures do not indicate any thresholds, and they change nonmonotonically with the collision energy displaying a local $\left(v_{i}, j_{i}\right)$-dependent maximum at low values of $E_{t r}$. The state-selectivity of the reaction is preserved. 


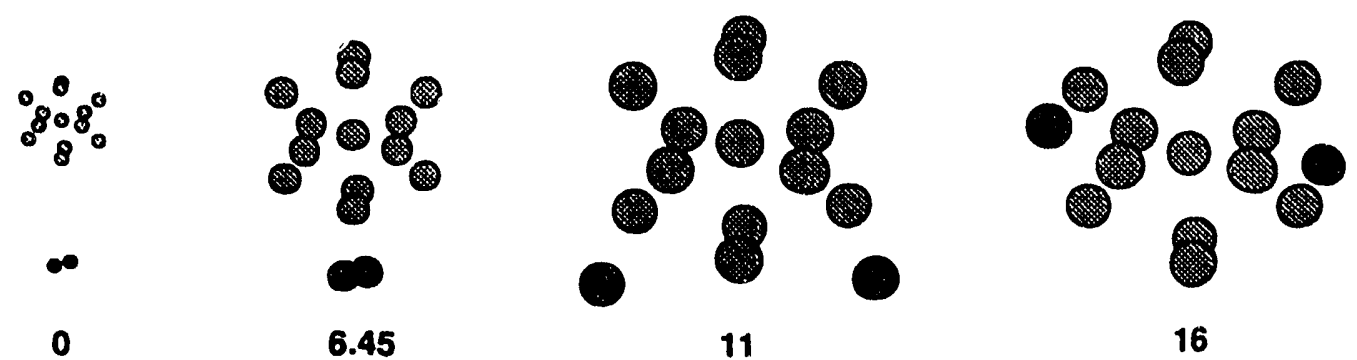

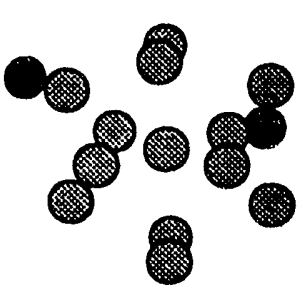

18

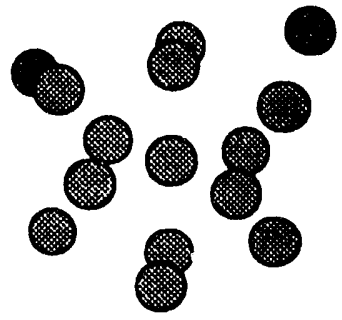

28

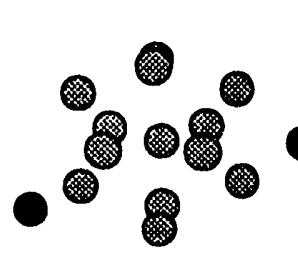

36

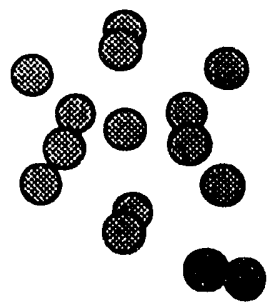

49

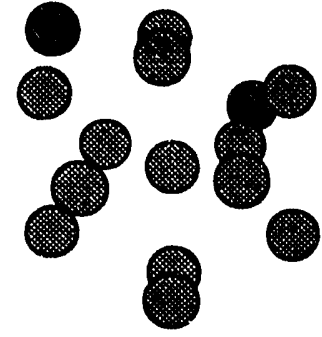

20

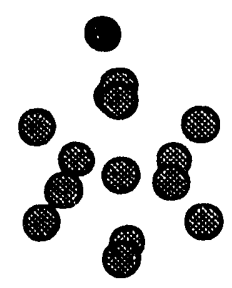

23

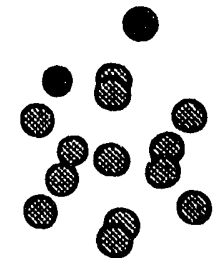

25

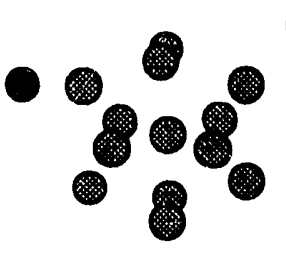

30

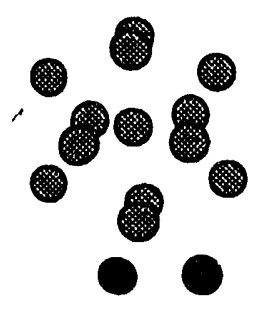

42

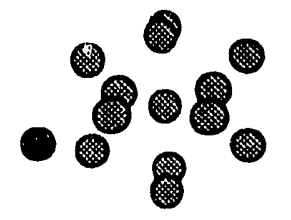

34.5 


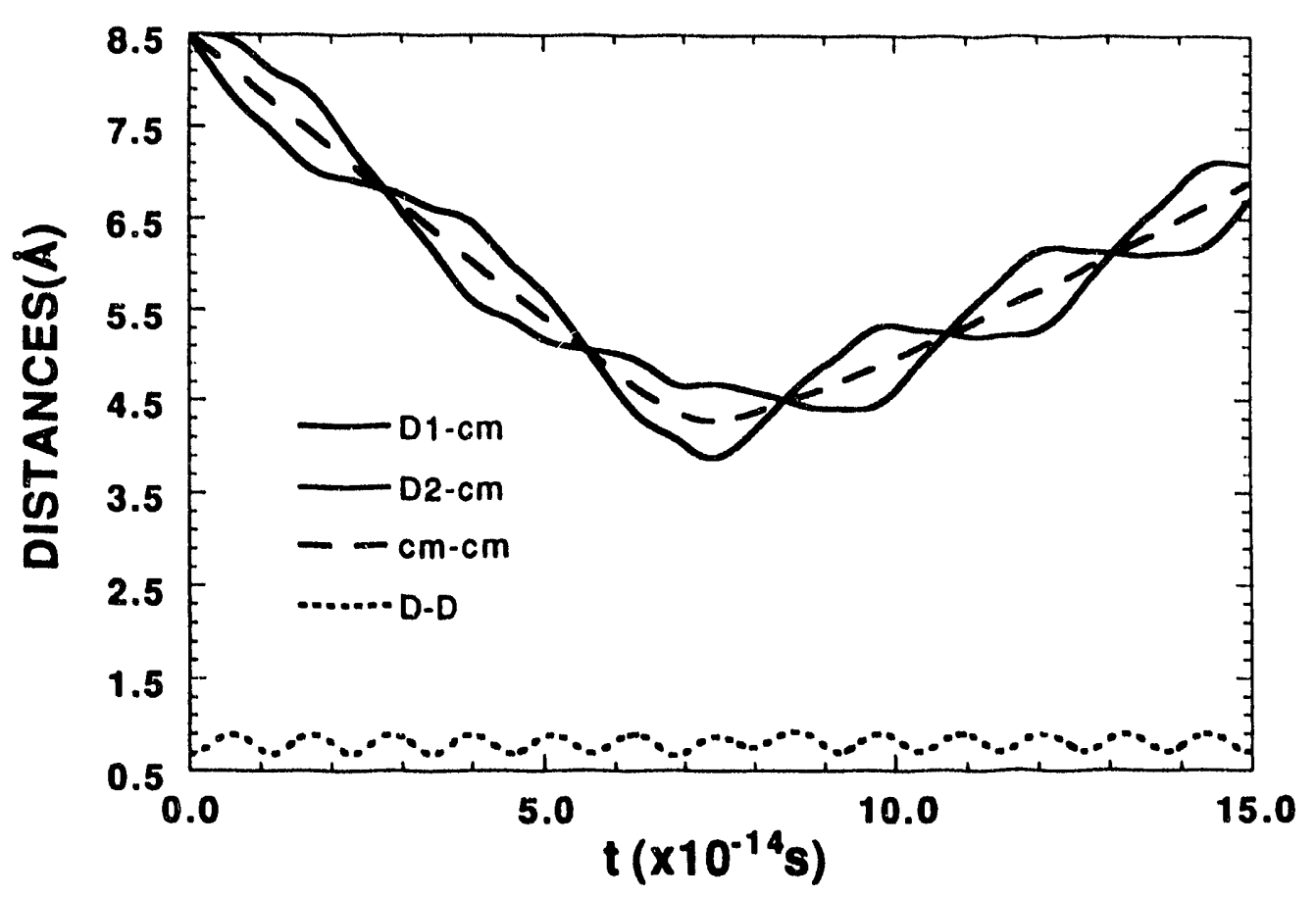

Figure 3. Characteristic distances (see text) as functions of time for the case of an inelastic scattering of $D_{2}\left(v_{i}=0, j_{i}=10\right)$ with a $N_{13}(i c 0, T=298 K) ; b=0.75 A ; E_{t r}=0.8 \mathrm{eV}$. The scattered molecule is departing in the ground vibrational state and carrying 13 quanta of rotational energy.

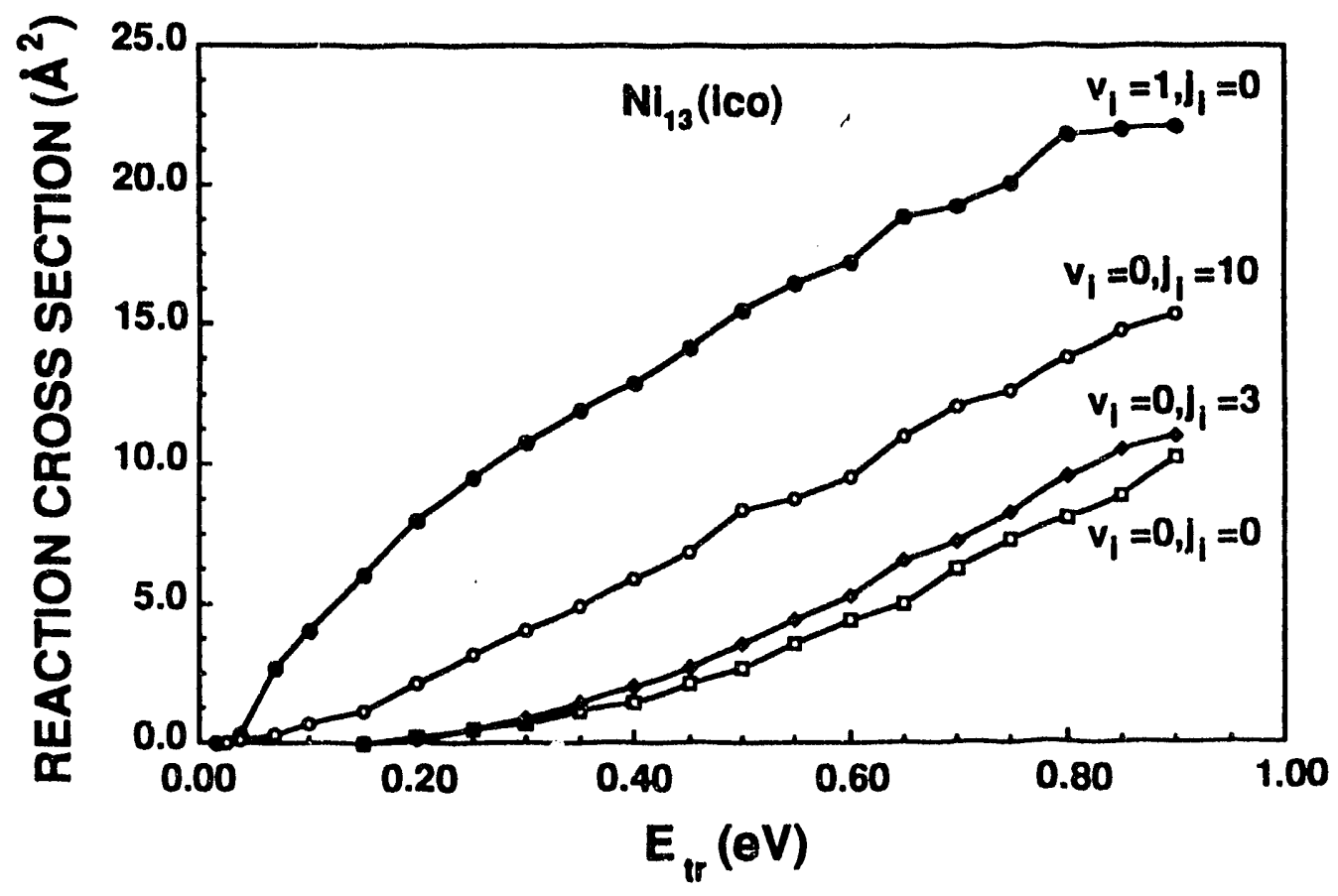

Figure 4. Reaction cross sections as functions of the collision energy for an ico $\mathrm{Ni}_{13}$ at $\mathrm{T}=0-298 \mathrm{~K}$ and a $D_{2}$ molecule in different initial rovibrational states. 


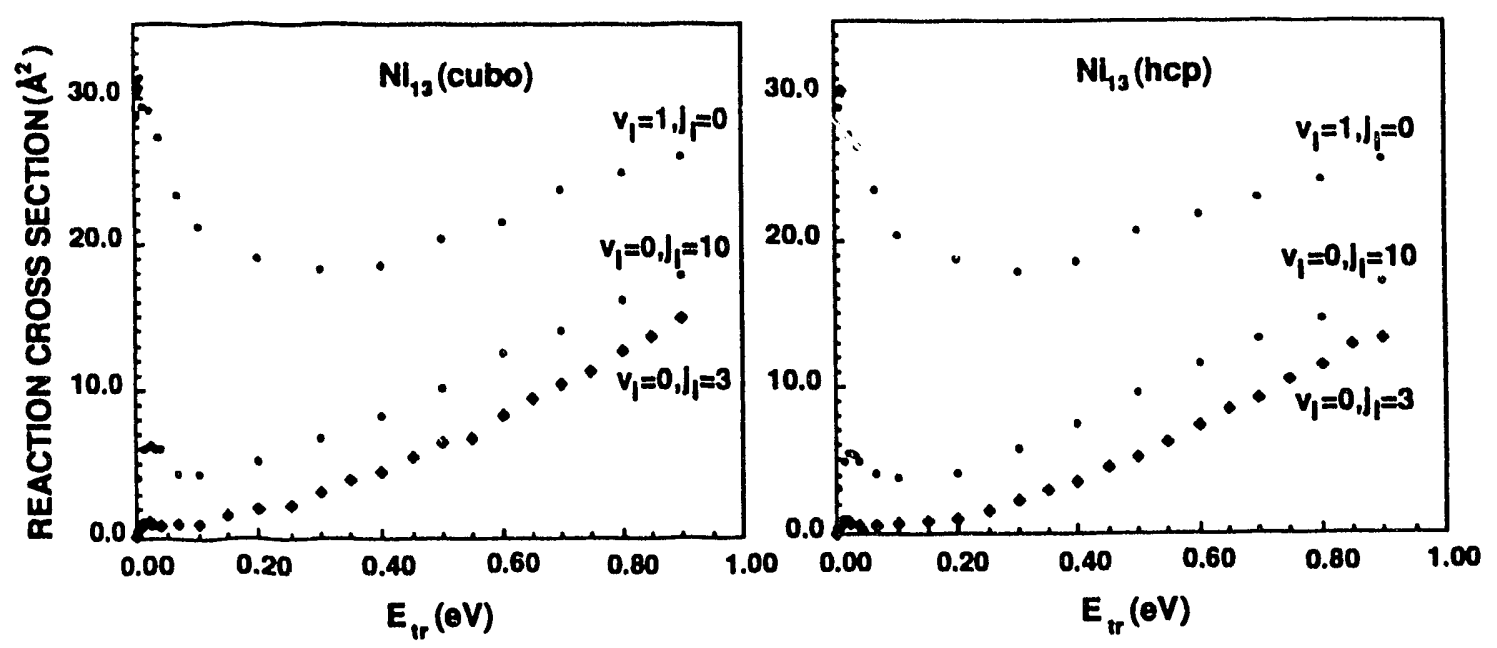

Figure 5. The same as Figure 4 but for zero-temperature cubo and hcp $\mathrm{Ni}_{13}$.

The fact that the major difference in the reaction cross sections is at low collision energies indicates that this difference is a consequence and reflection of a structurereactivity correlation. Indeed, it is at low values of $E_{t r}$ that the molecule spends longer times in the vicinity of the cluster surface and has a chance to explore and to "feel" its topology. The cubo and hcp isomers of $\mathrm{Ni}_{13}$ have very similar structures with surfaces formed by threefold and fourfold faces, whereas the surface of the ico isomer has only threefold faces. Using the analysis scheme described in the previous section, we determined that the low-energy maxima in the reaction cross sections are almost exclusively due to formation of reactive resonances. As the collision energy increases, the cross section of formation of these resonances, and therefore the cross section of the reaction, decreases. At still higher values of $E_{\mathrm{tr}}$ the direct pathway for the dissociative adsorption of the molecule begins to play an increasing role, and the reaction cross section becomes an increasing function of the collision energy. Table 1 contains data characterizing the reactive resonance lifetimes and the contribution of the direct and indirect pathways to the reaction. Whereas the fractional contribution of the direct reaction increases, the characteristic reactive-resonance lifetime decreases with an increase of $E_{t r}$. It should be noted that the absence of low-energy peaks in the reaction cross sections for the ico structure of $\mathrm{Ni}_{13}$ does not mean that the $D_{2}$ molecule does not form complexes with this isomer of the

Table 1. Probabilities POP and PIR of the direct and indirect reaction, respectively, the fractional contribution $f$ ? $)$ of the direct reaction, and reactive resonance lifctimes $\tau$... for a cubo $\mathrm{Ni}_{13}(\mathrm{~T}=0 \mathrm{~K})$, $D_{2}\left(v_{i}=1, j_{j}=0\right), b=0.25 \AA$ and different values of $E_{t r}$.

\begin{tabular}{|c|c|c|c|c|}
\hline$E_{t}(\theta V)$ & 0.038 & 0.30 & 0.50 & 0.90 \\
\hline PDR & 0.06 & 0.11 & 0.13 & 0.20 \\
\hline P.:. & 0.44 & 0.39 & 0.52 & 0.57 \\
\hline$f^{\mathrm{DR}}(\%)$ & 12 & 22 & 19 & 26 \\
\hline$\tau \ldots(p s)$ & 0.07 & 0.03 & 0.02 & 0.01 \\
\hline
\end{tabular}


cluster. It means rather that the low- $E_{t r}$ resonances involving this isomer are nonreactive, and they decay via the desorption of the molecule rather than its dissociation.

Because of the metastability of the cubo and hcp forms of $\mathrm{Ni}_{13}$, the temperaturedependence of the reactivity of the cluster was studied only for its ico isomer. The results for the case $\left(v_{i}=0, j_{i}=3\right)$ are displayed in Fig. 6 . The reactivity practically does not change in the temperature range $\mathrm{T}=0-298 \mathrm{~K}$. One obtains slightly higher reaction cross sections by heating the cluster to $600 \mathrm{~K}$. But then again, change of the cluster temperature in the range $T \approx 600-1450 \mathrm{~K}$ has almost no effect on its reactivity. As determined from the embedded-atom potential, the temperature range considered is below the value abcve which the cluster can be viewed as melted 20 . As seen, the reactivity of the cluster is at most weakly sensitive to varying its temperature in this range. Work is in progress to determine how further heating of the cluster, which will cause its melting, will affect its reactivity.

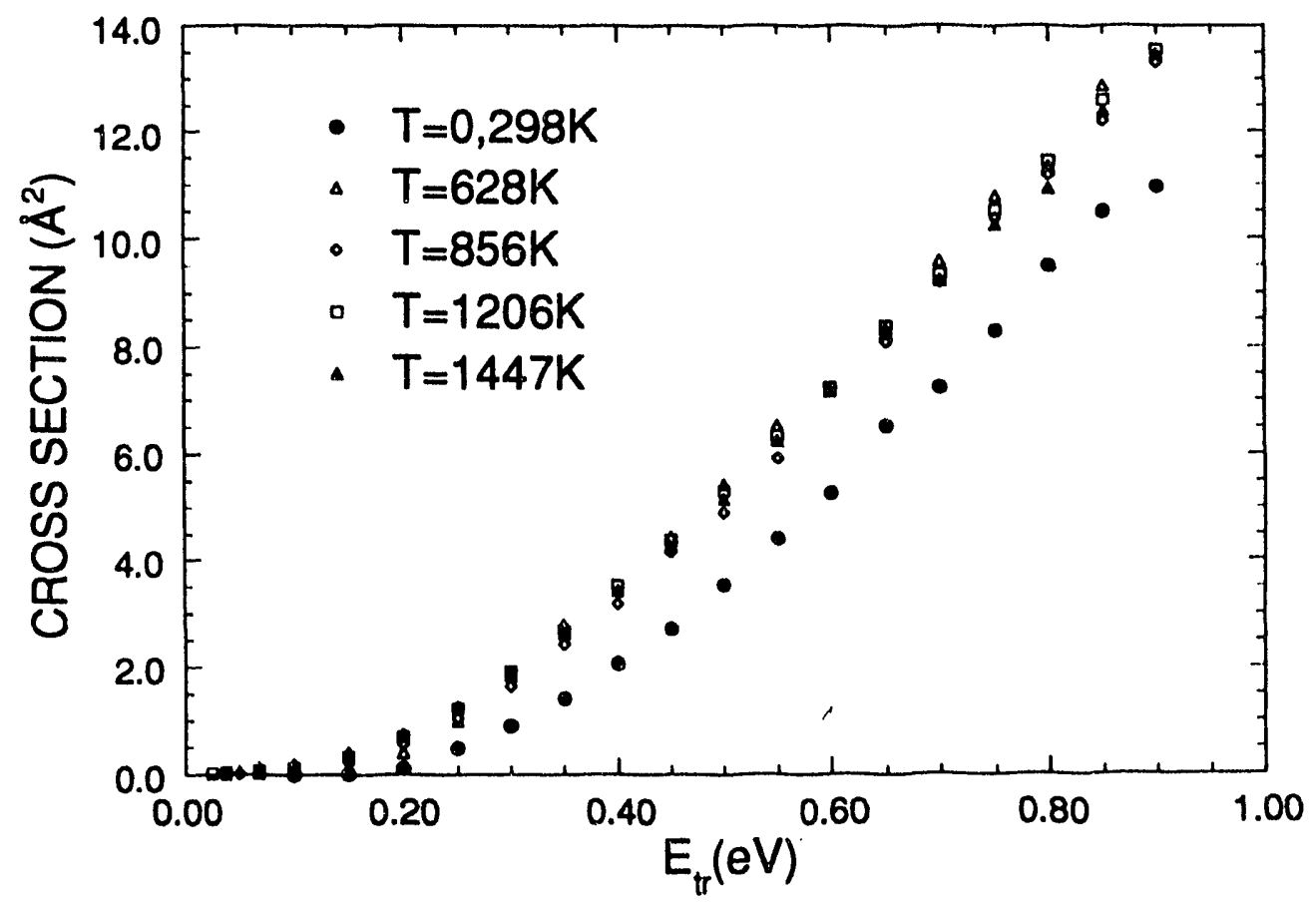

Figure 6. Reaction cross sections for $\mathrm{Ni}_{13}(\mathrm{ico}, \mathrm{T})+\mathrm{D}_{2}\left(\mathrm{v}_{\mathrm{i}}=0, \mathrm{j}_{\mathrm{i}}=3\right)$ as functions of the collision energy for different values of the cluster temperature $T$.

Our predictions $12,13,15$ regarding the important role of resonances as precursors in cluster-molecule reactions at low energies and the weak dependence of the cluster reactivity on its temperature ${ }^{11}$ (in the range considered) are consistent with recent experimental findings ${ }^{21,22}$. The value of the rate constant for the dissociative adsorption of a $D_{2}$ on a $\mathrm{Ni}_{13}$ inferred from room-temperature experiments ${ }^{22}$ is, however, considerably higher than the corresponding calculated value we obtained using the PESII parameterization of $V_{\text {LEPS: }}$ cf. also Ref. 9. The discrepancy indicates that the cluster-molecule interaction potential needs "tuning". The experimentally determined energy of binding of a D atom to the (111) and (100) faces of the bulk nickel ${ }^{23}$ is $2.74 \mathrm{eV}$, which is well reproduced by electronic structure calculations ${ }^{7,8}$. Using PESII we have calculated the binding energy of a $D$ atom adsorbed on a threefold face of an ico $\mathrm{Ni}_{13}$ cluster to be $2.43 \mathrm{eV}$. Although no experimental value for this quantity is available at present, arguments were forwarded ${ }^{8}$ in 
favor of it being close to or even exceeding the corresponding value for the D-surface binding. Guided by these considerations we increased the well depth of the Morse function describing the $\mathrm{D}-\mathrm{Ni}$ interaction in PESII, and thereby the $\mathrm{D}-\mathrm{Ni}_{13}$ binding energy, by $10 \%$. Using the thus modified ("tuned") $V_{\text {LEPS }}$ we recalculated the reaction rate constant corresponding to $\left(\mathrm{v}_{\mathrm{i}}=0, \mathrm{j}_{\mathrm{i}}=3\right)$ and (translational) temperature of $298 \mathrm{~K}$. The result is $3 \times 10^{-10} \mathrm{~cm}^{3} / \mathrm{s}$ which agrees well with the experimental estimate 22 of $(8 \pm 4) \times 10^{-10} \mathrm{~cm}^{3} / \mathrm{s}$.

The outcome of the collision of the molecule with the cluster is ultimately defined by the topology of the potential energy surface $V$ [Eq. (1)], which in our case is 39 dimensional. It may appear as impractical to attempt to analyze this surface and to try to understand how the "tuning" altered it. The problem, however, reduces to a much simpler one through the realization that binding of $\mathrm{D}_{2}$ molecule or of two separated $\mathrm{D}$ atoms to an ico $\mathrm{Ni}_{13}$ practically does not change the structure of the cluster. This justifies considering the cluster degrees of freedom as frozen and considering only the VLEPS part of $V$ which now depends on the remaining six degrees of freedom corresponding to the two $D$ atoms. These degrees of freedom can be chosen as the distance from the $\mathrm{cm}$ of the molecule to the $\mathrm{cm}$ of the nonmoving cluster, the D-D distance, and four angles (two for each distance) specifying the orientation of the corresponding separation vectors in the space. To characterize the phenomena considered we need only the distance degrees of freedom. Consequently, we have frozen the ico $\mathrm{Ni}_{13}$ in its equilibrium structure and minimized the PESII and the "tuned" versions of $V_{\text {LEPS }}$ over the four angular degrees of freedom. The result is two two-dimensional potential energy surfaces which depend on the relevant distances and which can be conveniently presented and analyzed in terms of equipotential contour plots. The details can be found in Ref. 16. In Fig. 7 the minimum energy paths corresponding to these surfaces are shown as a function of the D-D separation together

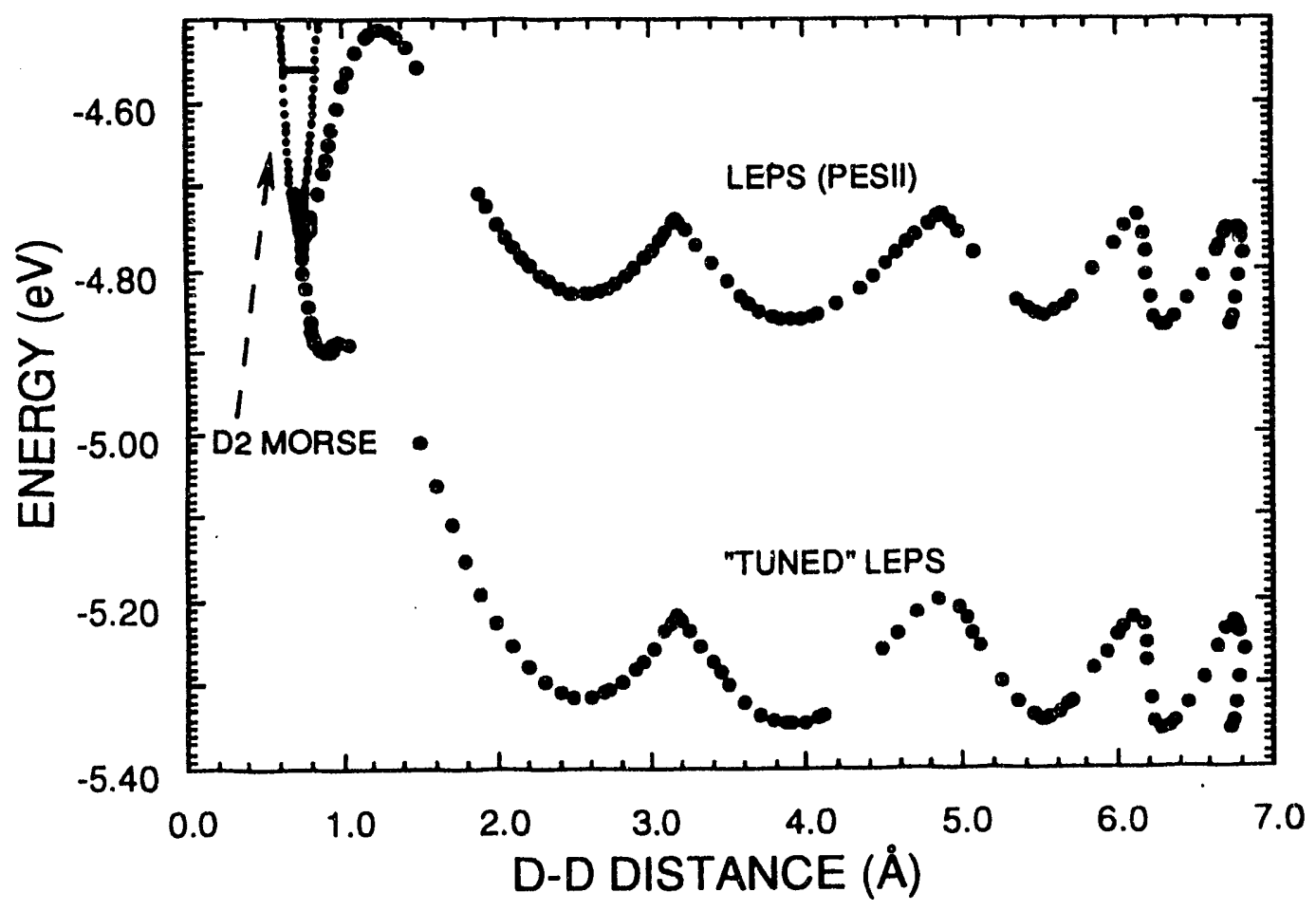

Figure 7. The minimum energy paths corresponding to the PESIl and the modified ("tuned") parameterizations of V LEPS (see text) as functions of the D-D distance. The Morse potential and the zeropoint energy of the $D_{2}$ molecule are also shown. 


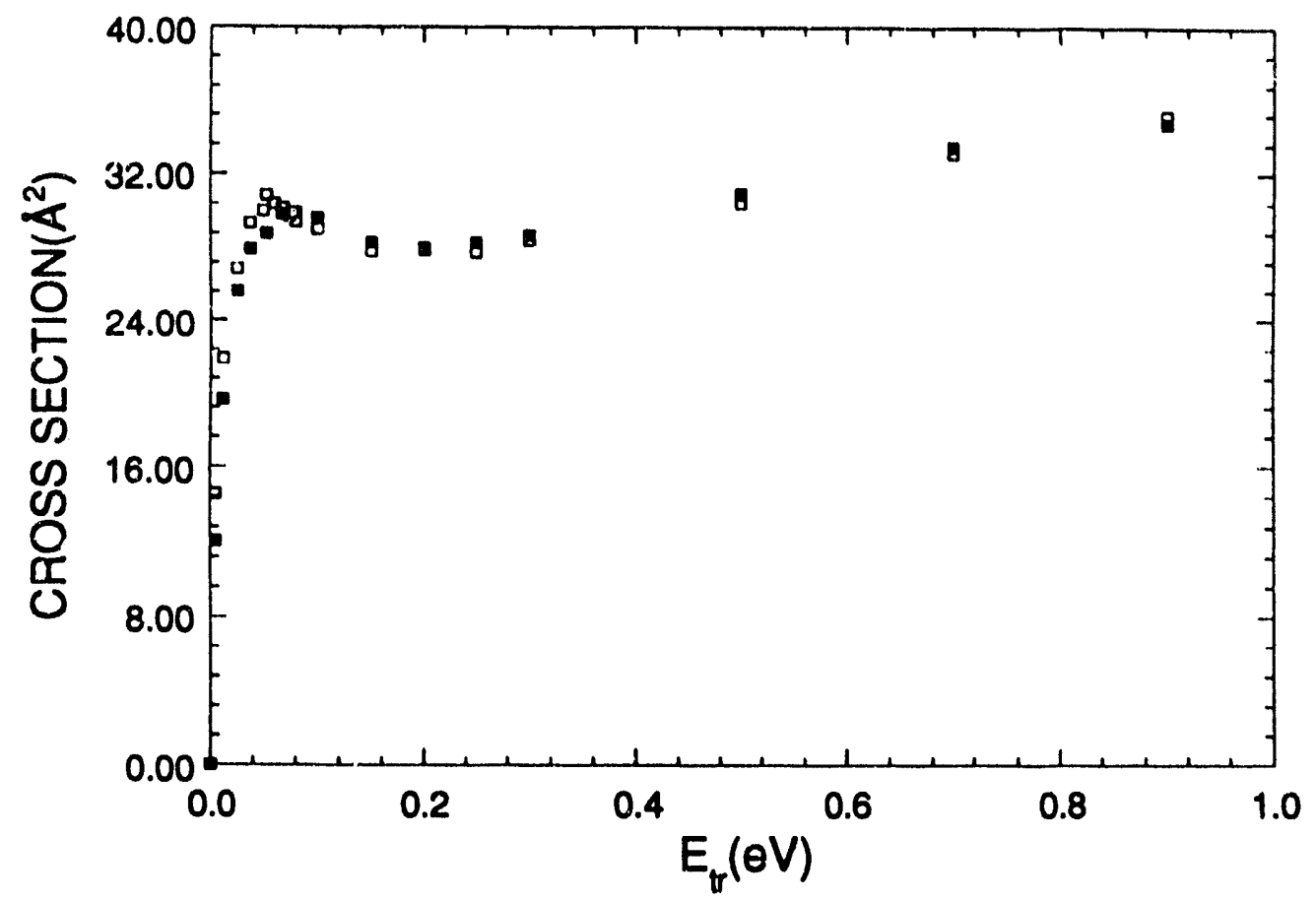

Figure 8. Reaction cross sections for $\mathrm{Ni}_{13}$ (ico, $\mathrm{T}=298 \mathrm{~K}$ ) and $\mathrm{Ni}_{13}$ (cubo, $\mathrm{T}=0 \mathrm{~K}$ ) [full and empty squares, respectively] calculated using the "tuned" $V_{\text {LEPS. The reactant }} D_{2}$ molecule was prepared in the $\left(v_{i}=0, j_{i}=3\right)$ state.

with the Morse potential and the zero-point energy of the $D_{2}$ molecule. The first minimum on the lhs in each of the LEPS curves is the well corresponding to the molecular adsorption. It is followed by a barrier to dissociation of the adsorbed molecule. The five additional wells corresponding to large D-D distances represent the five possible topologically different arrangements for the dissocjative adsorption. There are five topologically different pairs of threefold faces on an ico $\mathrm{Ni}_{13}$ which can serve as sites for adsorption of the separated $D$ atoms. The well with the largest $D-D$ separation corresponds to the case when the $D$ atoms are adsorbed on the opposite sides of the cluster. The maxima separating the dissociative adsorption wells are the diffusion barriers. They correspond to one of the $D$ atoms being over a face and the other over an edge of the cluster. The comparison of the two LEPS graphs clearly shows that, apart from a $10 \%$ parallel shift, the "tuning" has no effect on the minimum energy path in the "products" channel. It, however, drastically reduces the barrier to the dissociative adsorption. In fact, the molecule does not feel any barrier at all since the peak value of this barrier is below not only the level of the zero-point energy of $D_{2}$ but even that of the bottom of the $D_{2}$ Morse potential. It is this reduction in the reaction barrier that is responsible for a considerable enhancement of the cluster reactivity. In fact, the difference in the pattern of the reaction cross sections and the higher reactivity of the cubo and hcp isomers, as compared to the ico structure, which was discussed above, are also due to the difference in the heights of the corresponding reaction barriers as defined by the PESII parameterization of $V_{\text {LEPS }}$. Whereas for the ico $\mathrm{Ni}_{13}$ the peak of the barrier is above the level of the zero-point energy of $D_{2}$ (cf. Fig. 7), for the cubo and hcp isomers the corresponding peaks are below this level (details will be presented elsewhere).

It is of interest to inquire about the further implications of the performed modification

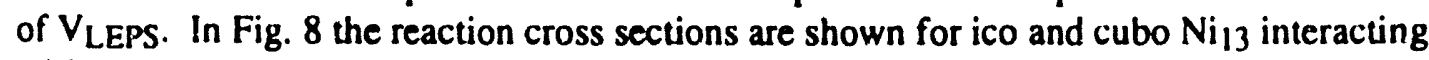

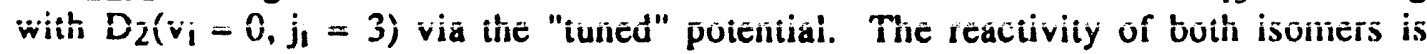
considerably higher than in the case of the PESII parameterization of V $V_{\text {LEPS }}$ (cf. Figs. 4 
and 5). The change in the cross section for the ico isomer is more dramatic: the peak at low collision energies indicates that with the modified $V_{\text {LEPS }}$ this isomer also can support reactive resonances. But perhaps the most striking is the almost total (except at very low collision energies) disappearance of the dependence on the cluster structure. In order to compare further the two LEPS surfaces, we have caiculated the frequencies of vibration along the $\mathrm{D}$-cluster and the $\mathrm{D}_{2}$-cluster bonds for the case of adsorption on a (threefold) face of an ico $\mathrm{Ni}_{13}$. In the case of atomic adsorption the frequencies are $754 \mathrm{~cm}^{-1}$ - for the PESII form of V LEPS, and $791 \mathrm{~cm}^{-1}$ - for the "tuned" potential. The two values are close. For an $\mathrm{H}$ atom they translate into $1066 \mathrm{~cm}^{-1}$ and $1123 \mathrm{~cm}^{-1}$, respectively. Eoth values agree well with the measured $24\left(1122-1137 \mathrm{~cm}^{-1}\right)$ and calculated ${ }^{7-3}\left(1043-1176 \mathrm{~cm}^{-1}\right)$ frequencies of vibration of an $\mathrm{H}$ atom over a threefold site of the $\mathrm{Ni}(111)$ surface. In the case of molecular adsorption the PESII gives $138 \mathrm{~cm}^{-1}$, whereas the modified VLEPS $382 \mathrm{~cm}^{-1}$. These two frequencies are sufficiently different to allow, at least in principle, for an experimental test of the two (or, possibly, more) parameterizations of the clustermolecule interartion energy. The difference in the predictions regarding the dependence of the cluster reactivity on its structure can, in principle, be used for this purpose as well.

\section{SUMMARY}

We have presented selected results of a detailed quasiclassical simulation study of processes induced by a collision of a $\mathrm{D}_{2}$ molecule with a $\mathrm{Ni}_{13}$ cluster. The focus was on the reactive channel, i.e., on the dissociative adsorption of the molecule. The dependence on factors such as the collision energy, the initial (quantized) rovibrational state of the molecule, and the structure and temperature of the cluster were analyzed. The direct and indirect (involving formation of transient complexes - resoniances) reaction pathways were considered and characterized quantitatively. We have illustrated how multidimensional potential energy surfaces describing cluster-molecule interactions can be analyzed in terms of a reduced set of relevant degrees of freedom and the topologies of these surfaces correlated with the dynamical phenomena extracted from the calculations. The complex problem of "tuning" the cluster-molecule interaction potential using experimental data has also been addressed, and a specific example of such "tuning" has been given. It underscores the crucial role of the intimate interplay between theory and experiment, especially in the field of clusters where, because of the large number of degrees of freedom involved, first principle calculations are (and will remain) of limited feasibility. More generally, the analysis and the discussion presented show that the phenomena and problems relevant for cluster-molecule collisions - a new research area by all accounts have much in common with those encountered in nucleus-nucleus, atom-atom, atommolecule, and molecule-molecule collisions - research fields with a considerable history.

\section{ACKNOWLEDGEMENTS}

This work was supported by the U.S. Department of Energy, Office of Basic Energy Sciences, Division of Chemical Sciences, under Contract No. W-31-109-Eng-38.

\section{REFERENCES}

1. See, e.g.. "Physics and Chemistry of Small Clusters", P. Jena, B. K. Rao, and S. N. Khanna, eds., Plenum Press. New York (1987); "Microclusters," S. Sugano, Y. Nishina, and S. Ohnishi, eds.,

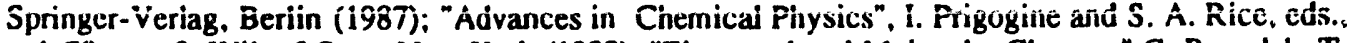
vol. 70, par 2, Wiley\&Sons, New York (1988); "Elemental and Molecular Clusters," G. Bencelck, T. 
P. Martin and G. Pacchioni, eds., Springer, Berlin (1988); "Physies and Chemistry of Finite Systems: From Clusters to Crystals," P. Jena, S. N. Khanna, and B. K. Rao, eds., Kluwer Academic Publishers. Dordrecht (1992); and references therein.

2. Sec. e.g., "Nuclear Physics Concepts in the Study" of Atomic Cluster Physics," R. Schmidt, H. O. Lutz, and R. Dreizler, eds., Springer, Berlin (1992).

3. See, e.g., W. Greiner and A. Sandulescu, Scientific American, March:58 (1990); and references therein.

4. J. Jellinek and D. H. Li, Phys. Rev. Lett. 62:241 (1989); D. H. Li and J. Jellinek, Z. Phys. D. 12:177 (1989); J. Jellinek and D. H. Li, Chem. Phys. Lett. 169:380 (1990).

5. Sec, e.g., J. L. Elkind, F. D. Weiss, J. M. Alford, R. T. Laaksonen, and R. E. Smalley, J. Chem. Phys. 88:5215 (1988); W. D. Reents, Jr. and M. L. Mandich, J. Phys. Chem. \$2:2908 (1988); S. A. Ruatta and S. L. Anderson, J. Chem. Phys. 89:273 (1988); W. F. Hoffman, E. K. Parks, and S. J. Rilcy. J. Chem. Phys. 90:1526 (1989); Y. M. Hamrick, and M. D. Morse, J. Phys. Chem. 93:6494 (1989); S. K. Loh, L. Lian, and P. B. Armentrout, J. Chem. Phys. $91: 6148$ (1989); S. Nonose, Y. Sonc, K. Onodera, S. Sudo, and K. Kaya, Chem. Phys. Lett. 164:427 (1989); P. Fayet, A. Kaldor, and D. M. Cox, J. Chem. Phys. 92:254 (1990); K. M. Creegan, and M. F. Jarrold, J. Am. Chem. Soc. 112:3768 (1990); R. E. Leuchtner, A. C. Harms, and A. W. Castleman, Jr., J. Chem. Phys. 92:6527 (1990); M. P. Irion and P. Schnabel, J. Phys. Chem. 95:10596 (1991); and references therein.

6. See, e.g., T. H. Upton, Phys. Rev. Lett. 56:2168 (1986); T. H. Upton, D. M. Cox, and A. Kaldor, in "Physics and Chemistry of Small Clusters", P. Jena, B. K. Rao and S. N. Khanna, cds., Plenum Press, New York (1987); K. Przybylski, J. Koutecky, V. Bonacic-Koutecky, P. von Ragué-Schleyer, and M. F. Guest, J. Chem. Phys. 94:5533 (1991); C. Mijoule, Y. Bouteiller, and D. R. Salahub, Surf. Sci. 253:375 (1991); and references therein.

7. See, c.g. P. E. M. Siegbahn, M. R. A. Blomberg, and C. W. Bauschlicher, J. Chem. Phys. 81:2103 (1984); I. Panas, J. Schule, P. Siegbahn, and U. Wahlgren, Chem. Phys. Lett. 149:265 (1988); E. A. Colbourn, Catalysis 8:42, Royal Society of Chemistry, Cambridge (1989) and references therein; U. Nielsen, D. Halstead, S. Holloway, and J. K. Nørskov, J. Chem. Phys. 93:2879 (1990); H. Yang, and J. L. Whitten; J. Chern. Phys. 98:4039 (1993); and references therein.

8. P. Mlynarski and D. R. Salahub, J. Chem. Phys. 95:6050 (1991).

9. K. Raghavan, M. S. Stave, and A. E. DePristo, Chem. Phys. Lett. 149:89 (1988); J. Chem. Phys. 91:1904 (1989).

10. J. E. Adams, J. Chem. Phys. $92: 1849$ (1990).

11. J. Jellinek and Z. B. Güvenç, Z. Phys. D. 19:371 (1991).

12. J. Jellinek and Z. B. Guvenc, in "Mode Selective Chemistry", p. 153, J. Jortner, R. D. Levine, B. Pullmann, eds., Kluwer Academic Publishers, Doidrecht (1991).

13. J. Jellinek and Z. B. Güvenc, in "Physics and Chemistry of Finite Systems: From Clusters to Crystals", vol. II, p. 1047, P. Jena, B. K. Rao, S. N. Khanna, eds., Kluwer Academic Publishers, Dordrecht (1992),

14. R. Foumier, M. S. Stave, and, A. DePristo, J. Cheın. Phys. 96:1530 (1992).

15. J. Jellinek and Z. B. Güvenç, in "Nuclear Physics Ccncepts in Atomic Cluster Physics, Lecture Notes in Physics", p. 169, H. O. Lutz, R. Schmidt, R. Dreizler, eds., Springer-Verlag, Heidelberg, (1992).

16. J. Jellinek and Z. B. Güvenç, Z. Phys. D 26:000 (194/3).

17. A. F. Voter and S. F. Chen, Mater. Res. Soc. Symy. 82:175 (1987).

18. T. N. Truong, D. G. Truhlar, and B. C. Garett, J. Phys. Chem. 93:8227 (1989).

19. R. N. Ponter, L. M. Raff, and W. H. Miller, J. Chern. Phys. 63:2214 (1975).

20. Z. B. Guvenç, J. Jellinek, and A. F. Voter, in "Physics and Chemistry of Finite Systems: Fıom Clusters to Crystals", vol. 1, p. 411 , P. Jena, S. N. Khanna, and B. K. Rao, eds., Kluwer Academic Publishers, Dordrecht (1992).

21. M. B. Knickelbein and W. J. C. Menezes, J. Phys. Chem. 96:6611 (1992); L. Zhu, J. Ho, E. K. Parks, and S. J. Riley, Z. Phys. D 26:313 (1993).

22. L. Zhu, J. Ho, E. K. Parks, and S. J. Riley, J. Chem. Phys. 98:2798 (1993).

23. K. Christmann, O. Schober, G. Ert, and M. Neuman, J. Chem. Phys. 60:4528 (1974).

24. W. Ho. N. J. DiNardo, and D. E. Pummer, J. Vac. Sci. Technol, 17:314 (1980); R. R. Cavanagh and R. D. Kelley. J. J. Rush, J. Chem. Phys. 77:1540 (1982). 

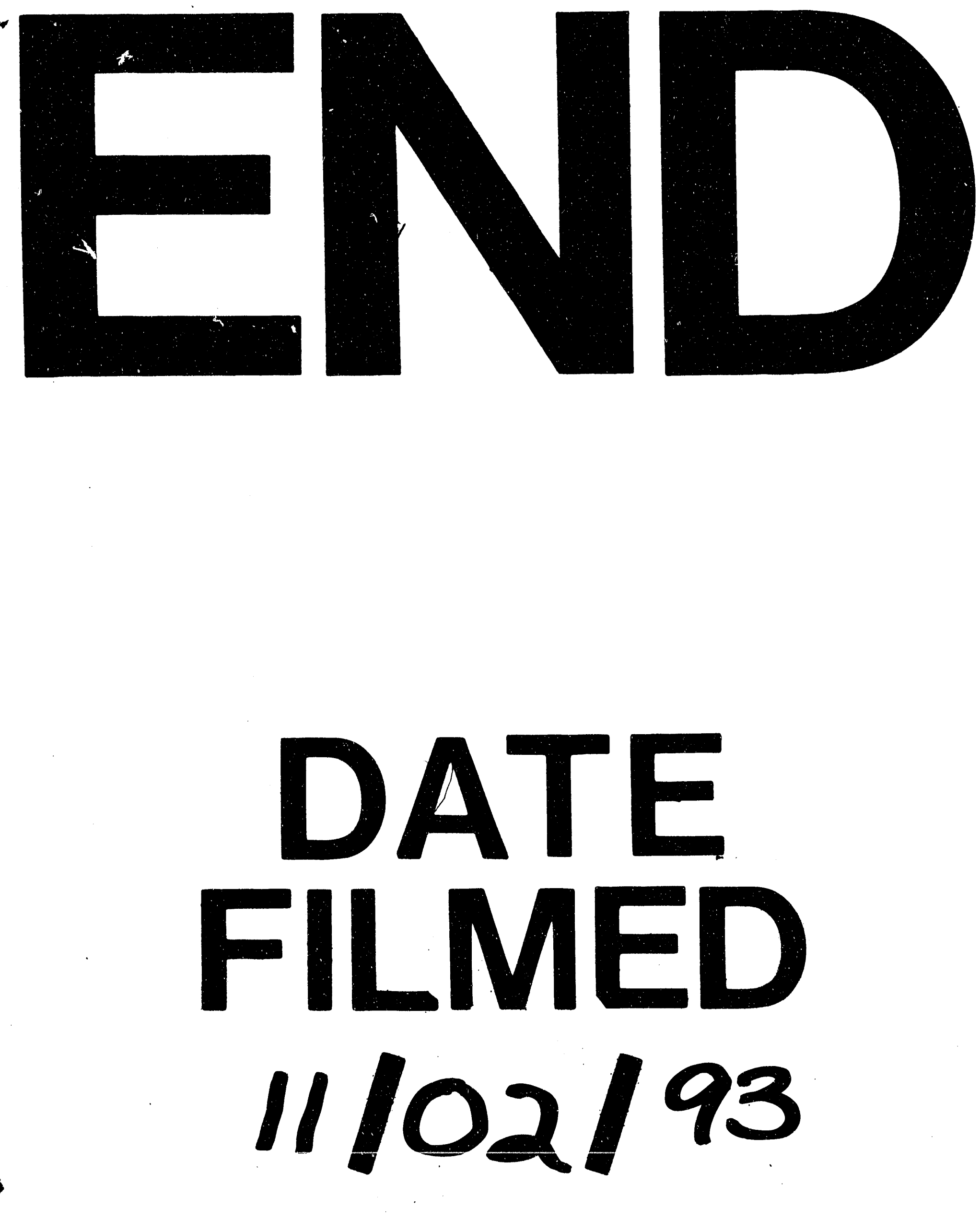
